\title{
An implantable CMOS signal conditioning system for recording nerve signals with cuff electrodes
}

\author{
Papathanasiou, Konstantinos; Lehmann, Torsten
}

Published in:

Proceedings on The 2000 IEEE International Symposium on Circuits and Systems

Link to article, DOI:

10.1109/ISCAS.2000.857419

Publication date:

2000

Document Version

Publisher's PDF, also known as Version of record

Link back to DTU Orbit

Citation (APA):

Papathanasiou, K., \& Lehmann, T. (2000). An implantable CMOS signal conditioning system for recording nerve signals with cuff electrodes. In Proceedings on The 2000 IEEE International Symposium on Circuits and Systems (pp. 281-284). IEEE. https://doi.org/10.1109/ISCAS.2000.857419

\section{General rights}

Copyright and moral rights for the publications made accessible in the public portal are retained by the authors and/or other copyright owners and it is a condition of accessing publications that users recognise and abide by the legal requirements associated with these rights.

- Users may download and print one copy of any publication from the public portal for the purpose of private study or research.

- You may not further distribute the material or use it for any profit-making activity or commercial gain

- You may freely distribute the URL identifying the publication in the public portal 


\title{
AN IMPLANTABLE CMOS SIGNAL CONDITIONING SYSTEM FOR RECORDING NERVE SIGNALS WITH CUFF ELECTRODES
}

\author{
Konstantinos Papathanasiou \\ Torsten L ehmann \\ Department of Information Technology, \\ $\emptyset$ rsteds Plads, Technical University of Denmark, \\ Building 344, DK-2800 Kgs. Lyngby, Denmark \\ kap@it.dtu.dk, tl@it.dtu.dk
}

\begin{abstract}
In this paper, we propose a system architecture for recording nerv e signals with cuff electrodes and we develop the key component in this system, the small-input, low-noise, low-pow er, high-gain amplifier. The amplifier is implemented using a mixture of weak- and strong-inversion transistors and a special off-set compensation technique; it's performance is validated using Spice simulations.
\end{abstract}

\section{INTRODUCTION}

Ev ery year thousands of individuals sustain a spinal cord injury, with a significant impact on the patien t'slife. Recent efforts in biomedical engineering address the issues of electronically monitoring and stimulating paralysed extremities $[1,2,3,4]$. These techniques are already used in volunteers to regain foot control of early multiple sclyroses patients and to assist the hand grip of tetraplegic persons [2].

Our involvement in this research is to develop implantable devices which will minimise the risk of infections by ensuring skin continuity [5]. In that way a bidirectional inductive link transmits power, con trol signals and feedback betw een the external control unit and the damaged nerve. This paper reports a subthreshold approach to biomedical signal amplification and nerve signal recording. An alternative approach to offset cancellation, with minimal impact at the input node, is presented which may find use in other amplifying schemes.

\section{IMPLANTED SYSTEM}

A special electrode is used in order to establish the necessary electrical contact with the nerve. This so called cuff electrode (figure 1) has a length of $10-20 \mathrm{~mm}$ and ph ysically connects the nerve to the implanted electronic device [1]. The electrodes are placed around the nerve by the use of local anaesthesia. The contact resistance after implantation is about $1 k \Omega$; the nerve how ev er gradually builds up a protective layer, which increases the resistance to $5 k \Omega$ [1] (aging process). The implanted device (figure 1) consists of an inductiv e loop around a silicon hip and some discrete components. The control signals and the pow er needed to operate the circuit are provided by an external control unit through the skin, by the use of an inductive link.

The implanted nerv e stim ulatorhas been reported elsewhere [5]. The feedbac $\mathrm{k}$ path must record very small $( \pm 10 \mu V)$, noisy nerve signals with very tight pow er requirements $(100 \mu A)$ posed by the induced pow er supply. The recording circuit facilitates the identification of the exited nerv e state, b y comparing the nere signal to the background activit $y$. Because we are not interested at the absolute value

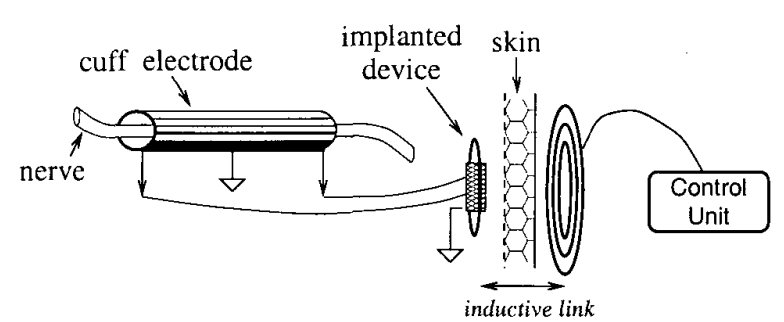

Figure 1. The ov erall implarted system

of the input signal, a $10 \%-15 \%$ variation of the gain or harmonic distortion can be tolerated.

Even if it $\mathrm{w}$ as possible to record the nerve signals with large accuracy, essentially we would be monitoring the thermal noise introduced by the cuff electrode. It therefore makes sense to perform some local signal processing in order to reduce the noise:

\section{NOISE AVERAGING}

The signal recovery technique $[1,2]$ is similar to demodulating an amplitude modulated signal. The input is amplified, an tialiased and sampled at $10 \mathrm{kHz}$. The digitised signal is rectified, low-pass filtered and downsampled thus the output results at a frequency of $20 \mathrm{~Hz}$, giving the en velope of the input nerve signal.

The variance of the nerve signal is:

$$
\sigma_{n}^{2}=\operatorname{Var}\left(V_{n}^{*}\right)=E\left\{\left(V_{n}^{*}-E\left\{V_{n}^{*}\right\}\right)^{2}\right\}=E\left\{\left(V_{n}^{*}\right)^{2}\right\}
$$

where $V_{n}^{*}$ and $\mu_{n}=E\left\{V_{n}^{*}\right\}=0$ are the (stochastic) nerve signal and its mean respectively.

The rectified signal $Y^{*}$ is given by the equation:

$$
Y^{*}=\sqrt{V_{n}^{* 2}} \text { and } E\left\{Y^{*}\right\}=E\left\{\sqrt{V_{n}^{* 2}}\right\}=\mu_{y}
$$

were $\mu_{y}$ is the mean of the rectified signal. The variance of $Y^{*}$ is given $\mathrm{b} y$ the equation:

$$
\begin{aligned}
\sigma_{y}^{2} & =E\left\{\left(Y^{*}-E\left\{Y^{*}\right\}\right)^{2}\right\}=E\left\{\left(\sqrt{V_{n}^{* 2}}-\mu_{y}\right)^{2}\right\} \\
& =E\left\{V_{n}^{-2}+\mu_{y}^{2}-2 \mu_{y} \sqrt{V_{n}^{* 2}}\right\} \\
& =\sigma_{n}^{2}+\mu_{y}^{2}-2 \mu_{y}^{2}=\sigma_{n}^{2}-\mu_{y}^{2}
\end{aligned}
$$

If the nerve signal is assumed to be Gaussian then we can find $\mu_{y}=\sqrt{2 / \pi} \cdot \sigma_{n}$ and $\sigma_{y}^{2}=(1-2 / \pi) \cdot \sigma_{n}^{2}$.

After a veraging the rectified signal the griance is reduced by $10 \mathrm{kHz} / 20 \mathrm{~Hz}$ and the overall output has a mean value 
and spread given $\mathrm{b} y$ :

$$
\mu_{y}=0.798 \cdot \sigma_{n}, \quad \sigma_{y}=0.027 \cdot \sigma_{n}
$$

where we have assumed uncorrelated samples and that the $v$ ariance does not change during the 500 samples. Thus we have a signal to noise ratio from the env elope detection procedure of $29 \mathrm{~dB}$. Such a resolution might seem small for signal processing tasks, how ever it is adequate to ealuate the state of a h uman nerve.

\section{THE PROPOSED ARCHITECTURE}

The overall system architecture is shown in figure 2 . A C coupling is used to minimise any DC offsets at the input (which can be hundreds of millivolts from the electrode). The $\pm 10 \mu \mathrm{V}$ input signal is subsequently amplified $10 \mathrm{k}$ times to a $\pm 100 \mathrm{mV}$ signal, whic hwill be antialiased by the use of a low-pass filter with a cut off frequency of $5 k \mathrm{~Hz}$. An Analogue to Digital Converter (ADC) is used to digitise the signal and the digital post-processing will include band-pass filtering [2], rectifying and averaging. The resultant samples will be transmitted by changing the load of the inductive link [6].

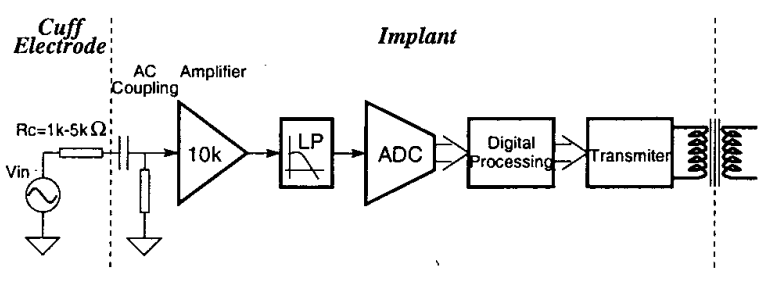

Figure 2. Overall circuit schematic

\section{ANTIALIASING}

As an antialiasing filter, w e use a single capacitor in conjunction with either the cuff electrode resistor or the second amplifier output resistance to achiev e a cut-off frequency of $5 k H z$ (se section 6.2.). Oversampling is used to suppress high frequency noise folded down in the signal band. From a power point of view, it often makes sense to $k$ eep lov signalto-noise ratio signal processing in the analogue domain [7]; how ever the signal processing done after the ati-aliasing filter can be made using very simple digital circuits, so in our case it makes sense to do the A/D conversion here. This also adds to the flexibility of the system.

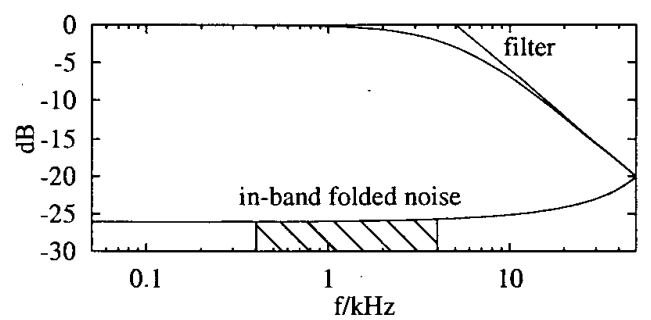

Figure 3. Aliasing effects

Our single pole, one capacitor filter has a $20 \mathrm{~dB} / \mathrm{Dec}$ response. Assuming a sampling frequency of $100 \mathrm{kHz}$ (10 times the Nyquist frequency), the normalised signal will be similar to that of figure 3. A t frequencies above $10 \mathrm{kHz}$ only the thermal noise of the input amplifier is present, because the nerve signals are negligible $[8,2]$ and the device is shielded by the surrounding tissue [8]. The aliased thermal noise which is folded down in the signal bandwidth $(400 \mathrm{~Hz}$ to $4 \mathrm{kHz})$ is atten uated more than $25 d B$ compared to the in-band noise, and is therefore not important.

\section{AMPLIFIER}

The noise introduced by the amplifier is of prime importance to signal integrity. Because of the minute signal from the electrode, it is crucial that it is amplified a lot immediately, using very few transistors (as these generate noise). The gate referred noise spectral densities of MOSFETs is approximately given by the equation:

$$
\frac{v_{i}^{2}}{\Delta f}=4 k T \frac{2}{3} \frac{1}{g_{m}}+\frac{K_{f}}{W L C_{o x} f}
$$

It is clear that the thermal noise can be reduced by increasing the transconductance $\left(g_{m}\right)$ of a transistor and that the flicker $(1 / f)$ noise can be reduced by increasing the transistor area $(W L)$. The transconductance of a MOSFET for the different modes of operation is given by:

$$
\text { subthreshold : } g_{m}=I_{d} /\left(n \cdot U_{T}\right)
$$

strong in version saturation : $g_{m}=2 \cdot I_{d} / V_{e f f}$

Where $U_{T}=26.7 \mathrm{mV}$ at $37^{\circ} \mathrm{C}$ (the typical body temperature), $n \simeq 1.2$, and the typical effectiv e voltage $V_{\text {eff }}=$ $V_{G S}-V_{T 0}=200 \mathrm{mV}$. Thus the transconductance to drain current ratio for a MOSFET is maximised in subthreshold operation and in our case it is about 3 times larger. Furthermore flicker noise is linked to surface effects in MOSFETs; therefore the transistor should exhibit low er $1 / f$ noise (smaller $K_{f}$ ) because subthreshold operation is dominated by diffusion current deep in the substrate $[9,10]$.

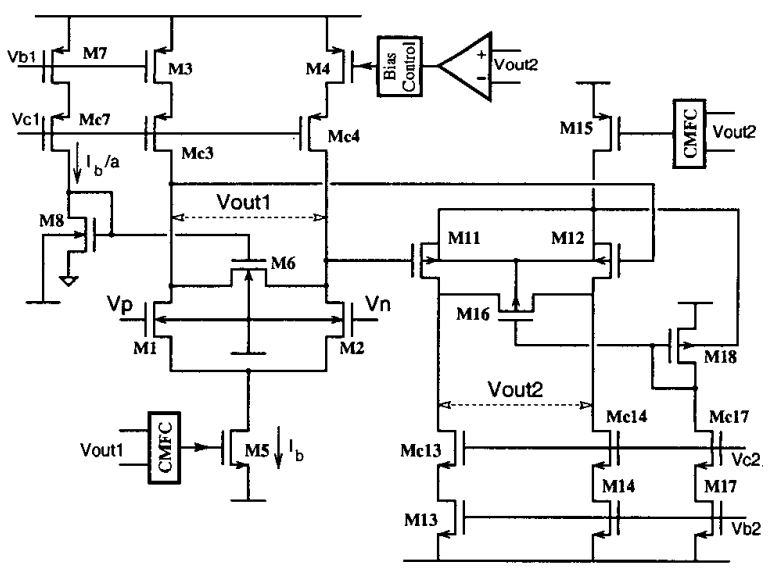

Figure 4. Amplifying stage

Ha ving these noise considerations in mind, propose the amplifier shown in figure 4 . The signal from the cuff electrode is AC coupled to the input transistors $\left(M_{1}, M_{2}\right)$ of the first stage to eliminate the DC offset in the electrode. The input transistors operate in subthreshold. The current needed to ensure low thermal noise is $35 \mu A$, a very large current for subthreshold operation; for this reason the NMOS input transistors should be designed to be very wide. The biasing current is supplied by the cascoded current sources $M_{3}, M_{c 3}$ 
and $M_{4}, M_{c 4}$. Those transistors operate in saturation to ensure good operation as current sources and to minimise their con tribution to noise [11]. A Common Mode F eedback Cir cuit (CMFC) is used to bias $M_{5}$ to stabilise the output common mode voltage to zero. $M_{6}$ operates in subthreshold and forms the load resistor which con trols the gain of the amplifier.

This gain is given by the equation:

$$
A_{i 1}=g_{m 1} \frac{r_{d s 6}}{2}=\frac{W_{1} / L_{1}}{W_{6} / L_{6}} \cdot \frac{1}{2 \cdot n}=100
$$

where $W_{i}, L_{i}$ the transistor width and length, n expresses the bulk effect and is given b y:

$$
\frac{1}{n}=1-\frac{\gamma}{2 \sqrt{V_{G}-V_{T 0}+\left(\gamma / 2+\sqrt{\Phi_{o}}\right)^{2}}} \simeq \frac{1}{1.2}
$$

were all the voltages are given with respect to the bulk voltage. Most of the $A_{v 1}$ mismatch in equation (1) is due to $n$ variations. How everif a maximum $30 \%$ variation in all $\gamma$, $V_{T 0}$ and $\Phi_{o}$ is assumed $n$ varies only by $5 \%$; well within a tolerable $\mathrm{v}$ ariation.

Equation (1) is valid pro vided that $V_{G 1} / n U_{T}-V_{S 1} / U_{T}=$ $V_{G 6} / n U_{T}-V_{S 6} / U_{T}$. The transistors $M_{7}, M_{8}$ generate $V_{G 6}$ to ensure that this assumption is valid:

$$
I_{d 3}=I_{d 7} \Rightarrow I_{d 01} \cdot e^{\frac{V_{G 1}-n V_{S 1}}{n U_{T}}}=I_{d 08} \cdot e^{\frac{V_{G 8}-n V_{S S}}{n U_{T}}}
$$

where $V_{G, S i}$ the gate, source voltage of transistor $i$ with respect to the bulk, $I_{d 01}=I_{d 08}$ is given in [7]. The CMFC ensures that $V_{S 8}=V_{D, S 6}=0 \mathrm{~V}$ and $V_{G 8}=V_{G 6}$ th us the assumption is valid and $A_{v 1}$ is given b y (1). In practice $I_{d 8}$ is a scaled down version of $I_{d 1}$, to reduce pow er consumption

The second amplifier has a similar topology with the first one, though all transistors operate in strong inv ersion because the signal levels here are too big for the subthreshold version. $M_{16}$ operates in the linear region and its effectiv e voltage defines the dynamic range of the amplifier. The noise of this amplifier referred to the input is divided by the gain $A_{v 1}^{2}$ therefore the bias current can be significantly reduced to $1 \mu A$. The gain of the second amplifier is and is given by the equation:

$$
A_{v 2}=g_{m 11} \frac{r_{d s 16}}{2}=\frac{W_{11} / L_{11}}{2 \cdot W_{16} / L_{16}}=100
$$

A $\mathrm{C}$ coupling bew een the $\mathrm{t} \mathrm{w}$ o amplifiers nabe considered, in order to minimise the effect that offsets might have at the output. In that way an overall gain of $10 k$ is realized in $t \mathrm{w} o$ stages with a gain of 100 each. Therefore the input signal is amplified from $\pm 10 \mu V$ to about $\pm 100 \mathrm{mV}$.

\subsection{Offset cancellation}

Offsets at the output of the amplifier can be easily removed, since we are only interested at the AC component of the nerve signal. How ever the task of cancelling the offset of the first stage is critical. The typical $V_{T 0}$ mismatch for a cen troid layout of the input transistors, in the process used, is more than an order of magnitude larger than the maximuminput signal. Suc h an offset will force the input transistors out of w eak in version in to saturation due to the high gain.

Switc hing the input will introduce charge feedthrough which will be much larger than theinput $v$ oltage and couple charge back to the nerve which can easily be damaged. Therefore an alternativ eoffset cancellation technique must be employed. Instead of using switc hestuning the current
$I_{d 4}$ is proposed. A continuous time bias control is applied to regulate the bias current by ensuring that the output of the second amplifier 4 is zero. In this case if the feedback loop is slow, the AC signal component is effectiv ely high-pass filtered. An alternative approach would require a current Digital to Analogue Converter (D A C) and a reset cycle to set the bias curren tin regular (relativ ely long) intervals to ensure zero output. This approach offers extended flexibility to the circuit. This offset cancellation has an effect of introducing curren $t$ mismatches,

$$
\Delta I_{1,2}=1-\frac{I_{1}}{I_{2}}=1-e^{\frac{V_{G 1}-V_{G 2}}{n \cdot U_{T}}}=3 \%
$$

However such a current mismatch will only generate offsets and will not affect the gain to a first order approximation.

Following the method presented above it is possible to realize accurate offset cancellation, without the use of switches which will introducing dynamic input offsets. It is this offset scheme that makes the implementation of such a subthreshold amplifier possible.

\subsection{Reducing pow er consumption}

The power is provided by the inductive link, therefore reducing the power consumption if of a prime importance. The most energy hungry device is the subthreshold amplifier, which uses $90 \%$ of the overall curren t to suppress the input noise. There are two ways of reducing pow er consumption of this amplifier

The first is to lower the pow er supply voltage for the first amplifier. This amplifier can operate with very low supply voltage: because the input transistors operate in subthreshold and the signal levels are very small, the overall $V_{d d}-V_{s s} \geq V_{T}-100 m V+2 V_{e f f} \simeq 1 V$.
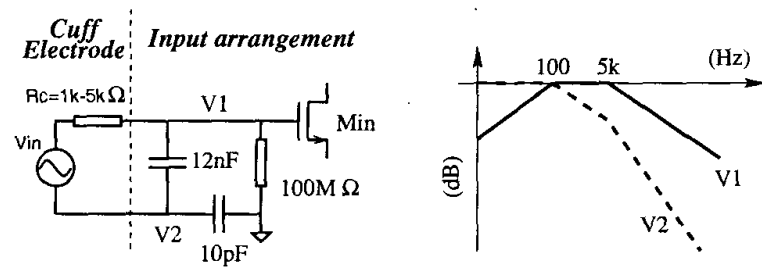

Figure 5. Input filter to reduce switching noise

An alternative and more pow er efficient way, is to lower the biasing currents of the subthreshold amplifier in between the sampling intervals. The amplifier needs $1 \mu s$ to settle down, therefore a small $5 \%$ duty cycle may be used, reducing the overall po w er consumption Nevertheless, when the biasing current on $M_{1}, M_{2}$ increases (to suppress the input thermal noise), $V_{S 1}=V_{S 2} \mathrm{~m}$ ust decrease (because $V_{G 1} \simeq V_{G 2} \simeq 0 \mathrm{~V}$ ), introducing charge feedthrough (through the relatively large parasitic capacitors $C_{G S 1}, C_{G S 2}$ ) and coupling charge back in the nerve.

The differential input scheme should minimise charge feedthrough effects and a modified input filter arrangement (figure 5) will significantly reduce this noise. This bandpass filter used for $A C$ coupling and antialiasing, ensures that the relatively large an tialiasing capacitance $(12 n F)$ provides a large charge reserwir, which holds the gate voltages at the gate of $V_{G S 1}, V_{G S 2}$ constant. (Note that when switching the bias current in the amplifier, the antialiasing filter must be placed before the amplifier; external components are necessary for this solution) 


\section{SIMULATION RESULTS}

A subthreshold amplifier has been simulated using the models of a $0.5 \mu \mathrm{m}$ process. Those simulations demonstrate the applicabilit y of the o verall design, with a view to do the loout of a chip to test the circuit. The biasing currents $I_{d 3}=I_{d 4} \mathrm{w}$ ere designed to $\mathrm{b} B 3.5 \mu \mathrm{A}$ with an effective voltage of $300 \mathrm{mV}$; this enables the current sources to be switched down to $1 \mu \mathrm{A}$, for saving power, with an effective voltage of about $50 \mathrm{mV}$. $I_{6}=250 n A$ for a maximum input voltage. The gain of the amplifier is 103 and the frequency response is shown in figure 6 . The amplifier has a $3 d B$ cut-off frequency at $1.5 \mathrm{MHz}$. This is due to the large bias currents whic hare needed for thermal noise suppression.

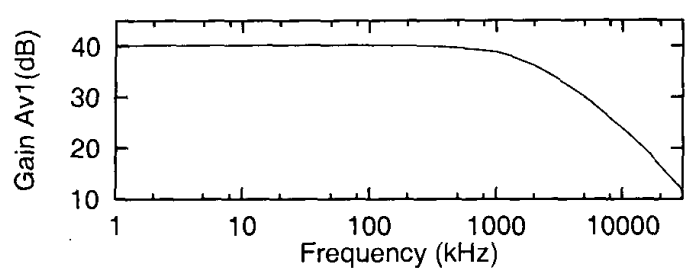

Figure 6. Subthreshold amplifier frequency response

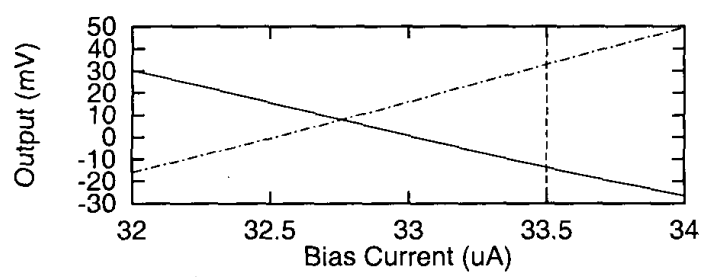

Figure 7. Current tuning for offset cancellation

The offset cancellation is shown in figure 7. A worst case $\Delta V_{T 0}=1 \mathrm{mV}$ between $M_{1}, M_{2}$ was introduced: This forces the output to become $50 \mathrm{mV}$. If the current $I_{d 4}$ is reduced to $32.7 \mu \mathrm{A}$ then this offset is cancelled. One can observe that the this cancellation can be made very accurate, as long as the bias con trol has a sufficien resolution. The current mismatch $\Delta I_{3,4}=2.4 \%$. Because te signal levels are so small, if the offset cancelling scheme is capable of keeping the amplifier at the correct bias point, distortion is much low er than what we can tolerate.

\section{CONCLUSIONS}

In this paper an implantable CMOS system for recording nerv e signals $w$ as investigated. This device will find use in restoring the mobility of human paralysed extremities. Because the signals are very small $( \pm 10 \mu V)$, the most critical component of the circuit is the amplifying stage. The use of subthreshold circuits, with large transconductance to current ratio, improved noise $c$ haracteristics and lw-v oltage properties, operating in a constant temperature environment $\left(37^{\circ} \mathrm{C}\right.$ body temperature), make our approach feasible for the extremely tight pow er requiremerts posed by the nature of the implantable device.

Relatively large offsets, distortion, or mismatches can be cancelled by the digital post processing algorithm. Nevertheless the implementation of such a subthreshold amplifier was made possible by ensuringhat an accurate threshold $\mathrm{v}$ oltage offset cancellation (without the use of switches which will introducing dynamic input offsets) was a vailable. A similar bias current tuning approach may be used for offset cancellation in different amplifying schemes.

The authors finally propose tw o curren $t$ reducing techniques, to further reduce power consumption. In that w ay more than one recording circuits may be used in an implantable device to enhance the flexibility of the device.

Presen tly test chips are being fabricated to experimentally verify the designs proposed in this paper.

\section{ACKNOWLEDGEMENTS}

Thanks are due to the Danish Technical Research Council for financial support.

\section{REFERENCES}

[1] M. Thomsen, Char acterisation and optimisation of whole nerve recording cuff electrodes. $\mathrm{PhD}$ thesis, Center F or Sensory-Motor Interaction, University of Aalborg, 1998.

[2] A. Lickel, R estontion of Lateral Hand Gr aspin a Trtraplegic Patient Applying Natural Sensory F eedback $\mathrm{PhD}$ thesis, Center For Sensory-Motor Interaction, University of Aalborg, 1998.

[3] D. B. Popovic, R. B. Stein, K. L. Jovano vic,R. Dai, A. Kostov, and W. W. Armstrong, "Sensory nerve recording for closed-loop control to restore motor functions," IEEE Transactions on Biomedical Engineering, vol. 40, no. 10, pp. 1024-1031, 1993.

[4] C. Enokawa, Y. Y oneza w.aH. Maki, and M. Aritomo, "Microcontroller-based implantable telemetry system for sympathetic nerve activity and ECG measurement," in A nnual International Confeence of the IEEE Engineering in Medicine and Biology, vol. 5, pp. 22322234, IEEE, 1997.

[5] G. Gundason, E. Brunn, and M. Haugland, "An implan table mixed analog/digital neural stimulator circuit," in International Symposium on Circuits and Systems, vol. 5, (Orlando), pp. 375-8, IEEE, 1999.

[6] Z. T ang, B. Smith, J. H. Schild, and P.H. P eckham, "Data transmission from an implantable biotelemeter by load-shift keying using circuit configuration modulator," IEEE Transactions on Biomedical Engineering, vol. 42, no. 5 , pp. 524-8, 1995.

[7] C. C. Enz and E. A. Vittoz, "CMOS low-pow er analog circuit design," in Emerging $T$ echnolo giesDesigning L ow Power Digital Systems (I. R. K. Cavin and W. Liu, eds.), pp. 79-133, IEEE, 1996.

[8] Q. Huang and M. Oberle, "A 0.5-mW passive telemetry IC for biomedical applications," IEEE Journal of SolidState Circuits, pp. 937-46, 1998.

[9] J. Chang, A. A. Abidi, and C. R. Viswanathan, "Flicker noise in CMOS transistors from subthreshold to strong inversion at $\mathrm{v}$ arious temperatures,"IEEE Transactions on Electron Devices, vol. 41, no. 11, pp. 1965-71, 1994.

[10] M. Aoki, H. Katto, and E. Yamada, "Low-frequency 1/f noise in MOSFETs at low curren tlevels," Journal of Applied Physics, vol. 48, no. 12, pp. 5135-40, 1977.

[11] K. G. Lamb, S. J. Sanchez, and W. T. Holman, "A low noise operational amplifier design using subthreshold operation," in Proceedings of Midwest Symposium on Circuits and Systems, pp. 35-8 vol.1, IEEE, 1998. 